\title{
Enhancement of Faraday rotation in defect modes of one-dimensional magnetophotonic crystals
}

\author{
TAhmineh Jalali ${ }^{1 *}$, AbDolrasoul GHaraAti $^{2}$, Mohammad RASTEGaR $^{3}$ \\ ${ }^{1}$ Physics Department, Persian Gulf University, Bushehr, Shahid Mahini Street, 75169-13817, Iran \\ ${ }^{2,3}$ Physics Department, Payame Noor University, Tehran, Artesh Blvd., 19395-4697, Iran
}

\begin{abstract}
In this paper, employing of one-dimensional magnetophotonic crystals in infrared wavelengths range is considered. For this purpose, magnetophotonic multilayer structures, composed of magnetic defect layer surrounded by dielectric and MO Bragg mirrors, have been proposed. Ce:YIG with an optical thickness in the range of 0 to $\lambda_{\mathrm{s}}$ was used as a magnetic material. By using four by four transfer matrix method, the transmittance values and Faraday rotation (FR) angles of these structures were computed. The electric field distribution was obtained by Finite Element Method (FEM). By investigation of transmittance and FR angle of magnetophotonic crystals, it was possible to design the optimized structures with a rotation larger than 30 degrees and high transmittance. Such structures with a few micrometer thickness and fast magneto-optical (MO) responses have the potential to be used in MO devices like integrated photonic elements and sensors.
\end{abstract}

Keywords: magneto-optics; magnetophotonic crystal; transmittance; Faraday rotation

\section{Introduction}

During the few past years, much attention has been paid to researching light propagation in photonic crystals [1-6]. These structures have a photonic band gap (PBG), where light cannot propagate. This feature is the basis for developing photonic crystal devices such as optical filters, switches, diodes, etc. The light can be localized by creating the cavities and waveguides in the photonic crystal structures [4-6]. Recently, one dimensional magnetophotonic crystals (1D-MPCs) consisting of periodic dielectric and anisotropic magnetic thin films have attracted much attention because of their particular properties, such as controlling light propagation by applying a magnetic field $[7,8]$.

Enhancement of Faraday rotation and transmittance at a localized mode or near the PBG edges has a fundamental importance [912]. The defects play an important role to increase the magneto-optical (MO) response [1220]. The magnetophotonic crystals with PBG, seem

\footnotetext{
*E-mail: jalali@pgu.ac.ir
}

to be ideal structures for fabrication of optical isolators and circulators, optical switches, light modulators and magnetic field sensors. The Faraday rotation (FR) effect is strongly enhanced in the magnetophotonic microcavities [10-12]. For 1D-MPCs, these features can be attributed to the enhanced FR of the resonant mode in the multilayer films. The rotation angle of magnetic monolayer is generally small. Therefore, we used 1D-MPCs with defect layers in the middle to enhance the rotation angle. A rotation angle close to 45 degrees can be achieved in the proposed structures by introducing defect layers with a total thickness of a few micrometers.

In this paper, we have discussed MO Faraday effect in the 1D-MPCs constituted of dielectric and magnetic materials in terms of theory and computation. The MO response of $1 \mathrm{D}-\mathrm{MPCs}$ have been studied through $4 \times 4$ transfer matrix method (TMM). We simulated the magnetophotonic multilayers and calculated their transmittance and FR angle. The rotation angles of 1D-MPCs were measured as a function of repetition number and thickness of the magnetic defect layer. In addition, the electric field distribution was obtained 
by finite element method (FEM) simulation. The analysis of the structures with MO Bragg mirrors is given in subsection 3.1. In case of these structures, the magnetic defect layer occurs in the middle of 1D-MPCs. The magnetophotonic multilayers with the magnetic defect layer surrounded by dielectric photonic crystals are investigated in subsection 3.2. Section 4 includes the summary of the research findings.

\section{Theory and model}

We have shown an example of magnetophotonic multilayer structure made of periodic thin films of dielectric (D) and magnetic (M) materials in Fig. 1. The 1D-MPC has periodicity and a magnetic defect layer located in the middle as $(\mathrm{DM})^{\mathrm{m}}(\mathrm{M})(\mathrm{MD})^{\mathrm{m}}$, where $\mathrm{m}$ denotes the repetition number. The FR angle of 1D-MPCs is evaluated by TMM. The optical field inside each layer is given as a sum of right and left circular polarized waves propagating in opposite directions and normal to the multilayer structure.

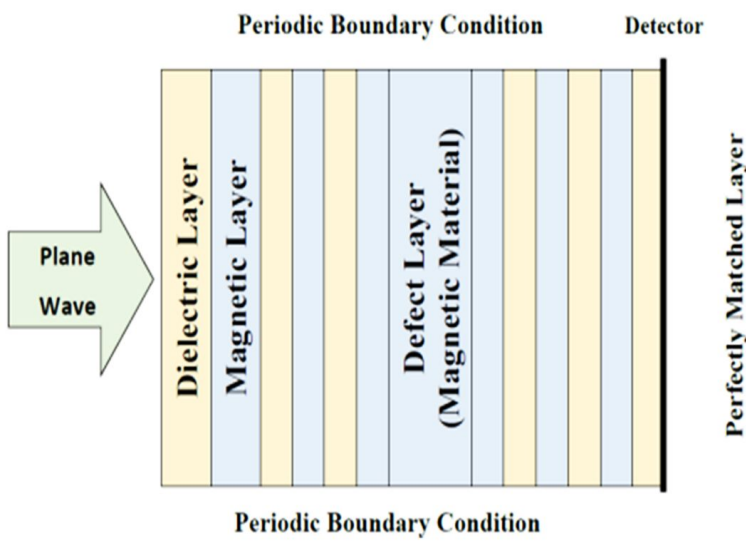

Fig. 1. Schematic structure of 1D-MPC composed of alternating layers of magnetic and dielectric materials described as $(\mathrm{DM})^{\mathrm{m}}(\mathrm{M})(\mathrm{MD})^{\mathrm{m}}$ with $\mathrm{m}=3$. The boundary conditions, plane wave and detector are shown.

According to Maxwell equation, the light propagates through a layered structure as:

$$
\nabla \times\left(\frac{1}{\mu} \nabla \times \vec{E}\right)-k_{0}^{2}\left(\frac{\varepsilon-i \sigma}{\omega \varepsilon_{0}}\right) \vec{E}=0
$$

where $\epsilon$ and $\mu$ are permittivity and magnetic permeability tensors of the medium, respectively. The light propagation in each layer is connected with optical properties of the medium. For a z-axis uniformly magnetized medium, the symmetry reduces to one axis. Therefore, the permittivity tensor of magnetic material is described as a sum of symmetric and asymmetric tensors in the following form $[21,22]$ :

$$
\begin{aligned}
\varepsilon_{M} & =\left(\begin{array}{ccc}
\varepsilon_{x x} & 0 & 0 \\
0 & \varepsilon_{y y} & 0 \\
0 & 0 & \varepsilon_{z z}
\end{array}\right)+\left(\begin{array}{ccc}
0 & -i g & 0 \\
i g & 0 & 0 \\
0 & 0 & 0
\end{array}\right) \\
& =\left(\begin{array}{ccc}
\varepsilon_{x x} & -i Q \varepsilon_{x y} & 0 \\
i Q \varepsilon_{x y} & \varepsilon_{y y} & 0 \\
0 & 0 & \varepsilon_{z z}
\end{array}\right)
\end{aligned}
$$

with

$$
\begin{gathered}
Q=\frac{\lambda}{\pi \cdot \sqrt{\varepsilon_{x y}}} \cdot V_{M} \cdot B_{z} \\
\varepsilon_{x y}=\frac{1}{2} \cdot\left(\varepsilon_{x x}+\varepsilon_{y y}\right)
\end{gathered}
$$

where $\varepsilon_{\mathrm{xx}}=\varepsilon_{\mathrm{yy}} \neq \varepsilon_{\mathrm{zz}}$, g is the magnetic gyration constant, $\mathrm{V}_{\mathrm{M}}$ is the Verdet constant and $\mathrm{Q}$ is the magneto-optical parameter, which is related to gyration constant of magnetic media, that is defined as $Q=\frac{\mathrm{g}}{\varepsilon_{\mathrm{xx}}}$. In a medium that is affected by a magnetic field, the rotation directions in a plane that is perpendicular to the magnetic field are different. Therefore, the non-diagonal elements appear in the permittivity tensor of the magnetic medium. This leads to a difference between the refractive indices of the right- and left-handed circularly polarized waves and then different velocities of light traveling in the magnetized medium. Based on the above considerations, the polarization plane of a linearly polarized wave rotates (Faraday effect) as [21]:

$$
\theta=\left(n_{+}-n_{-}\right) \frac{\omega L}{2 c}
$$

where $\mathrm{n}_{+}$and $\mathrm{n}_{-}$denote refractive indices of rightand left-handed circularly polarized waves, respectively. The permittivity of the dielectric layer is $\varepsilon_{D}$, which can be defined as $n_{D}=\sqrt{\varepsilon_{D}}$. For illuminating a multilayer magnetophotonic structure with 
polarized electromagnetic waves, the transfer matrix is defined as:

$$
T=D^{-1}(0) \cdot\left(\prod_{n=1}^{N} D(n) P(n) D^{-1}(n)\right) \cdot D(0)
$$

where $D(n)$ is the dynamical matrix of $n$-th layer. $\mathrm{P}(\mathrm{n})$ and $\mathrm{N}$ are the propagation matrix and number of layers, respectively. By considering the field continuity between the layers, we can use the following relation between the amplitudes of electric and magnetic fields in two adjacent layers [23]:

$$
\left(\begin{array}{l}
A_{1}(n-1) \\
A_{2}(n-1) \\
A_{3}(n-1) \\
A_{4}(n-1)
\end{array}\right)=D^{-1}(n-1) D(n) P(n)\left(\begin{array}{l}
A_{1}(n) \\
A_{2}(n) \\
A_{3}(n) \\
A_{4}(n)
\end{array}\right)
$$

where

$$
D(n)=\left(\begin{array}{llll}
\hat{x} \cdot p_{1}(n) & \hat{x} \cdot p_{2}(n) & \hat{x} \cdot p_{3}(n) & \hat{x} \cdot p_{4}(n) \\
\hat{y} \cdot q_{1}(n) & \hat{y} \cdot q_{2}(n) & \hat{y} \cdot q_{3}(n) & \hat{y} \cdot q_{4}(n) \\
\hat{y} \cdot p_{1}(n) & \hat{y} \cdot p_{2}(n) & \hat{y} \cdot p_{3}(n) & \hat{y} \cdot p_{4}(n) \\
\hat{x} \cdot q_{1}(n) & \hat{x} \cdot q_{2}(n) & \hat{x} \cdot q_{3}(n) & \hat{x} \cdot q_{4}(n)
\end{array}\right)
$$

with $\mathrm{q}_{\sigma}=\frac{\mathrm{c}}{\omega} \mathrm{k}_{\sigma} \times \mathrm{p}_{\sigma}(\sigma=1,2,3,4)[23,24]$. In this relation, $\mathrm{p}_{\sigma}$ and $\mathrm{q}_{\sigma}$ are the polarization vectors and $\mathrm{k}_{\sigma}$ is a wave vector. $\hat{\mathrm{x}}$ and $\hat{\mathrm{y}}$ are unit vectors along $\mathrm{x}$ and y directions. Finally, the FR angle $\theta_{\mathrm{F}}$ is given by [23]:

$$
\theta_{F}=\arg \left(\frac{T_{33}}{T_{11}}\right) \cdot \frac{90}{\pi}
$$

For simplest case, the rotation angle is proportional to the magnetic field $\mathrm{H}$ and distance $\mathrm{L}$ traveled by light in matter as [21]:

$$
\theta=V_{M} H L
$$

The Verdet constant of the magnetic medium $\mathrm{V}_{\mathrm{M}}$ is defined as rotation of polarization plane of light per unit path and magnetic field. The Verdet constant of the magnetized media can be computed from the following relation [21]:

$$
V_{M}=\frac{e}{2 m c^{2}} \lambda \frac{d n}{d \lambda}
$$

where e and $m$ are charge and mass of electron, respectively and $\lambda=\frac{2 \pi \cdot c}{\omega}$ is the wavelength of light.
This constant depends on the wavelength of incident wave, ambient temperature and properties of the medium. The transfer matrix calculation was described in detail by Kato [10].

\subsection{Principles of FEM}

In this method, the simulation and computation were made based on six steps: (1) Dividing the computational space into finite elements. (2) Introducing a function representing physical properties of each element. (3) Obtaining equations for each element. (4) Combining the equations of all elements and generating the equations system. (5) Applying the boundary conditions on the location of the element node. (6) Solving the equations system. The FEM formulation includes weighted residual methods and minimum potential energy. The problem has been solved by Euler method by eliminating the differential equations or simplifying them to usual equations. The Maxwell equations were solved by considering the boundary conditions and physical properties of the material. Using this technique, the electromagnetic waves in optical and photonic systems can be simulated. Optical devices such as waveguides, cavities and filters can be simulated and examined by FEM. To study the magnetophotonic structure, equation 1 was solved using the periodic boundary conditions:

$$
\hat{n} \times\left(E_{1}-E_{2}\right)=0
$$

$$
\hat{n} \times\left(H_{1}-H_{2}\right)=0
$$

Also, we have used the scattering boundary conditions in the form:

$$
\begin{aligned}
& \hat{n} \times(\nabla \times \vec{E})-i k \hat{n} \times(\vec{E} \times \hat{n}) \\
& =-\hat{n} \times\left(\vec{E}_{0} \times\left(\vec{k} \times\left(\hat{n}-i \hat{k}_{d i r}\right)\right)\right) \cdot \exp \left(-\hat{k}_{d i r} \cdot \vec{r}\right)
\end{aligned}
$$

where $E_{0}$ is incident electric field, $\hat{n}$ is the normal direction and $k_{\text {dir }}$ is the incident wave vector. The first step was related to the mesh structure and its conditions. The properties of mesh structure should enhance the accuracy of computations and reduce the use of memory. In fact, the balance 
between these two factors must be established. The simulated electric and magnetic fields were employed to calculate other optical parameters [25]. The overview of the boundary conditions of FEM simulation is shown in Fig. 1.

\section{Results and discussion}

\subsection{The structures with MO Bragg mir- ror}

The studied 1D-MPCs consist of Ce:YIG (cerium substituted yttrium iron garnet) as magnetic material and $\mathrm{SiO}_{2}$ or $\mathrm{TiO}_{2}$ as dielectric materials. The optical thickness of these layers is equal to one quarter of the wavelength. We have used Ce:YIG as the magnetic material due to the low absorption in the infrared region and high MO responses. This issue is attractive for practical applications and experimental studies. The magnetic Ce:YIG layer has the diagonal permittivity $\varepsilon_{\mathrm{X}}=4.884$ and the non-diagonal permittivity of (0.009 i) at $\lambda=1.55 \mu \mathrm{m}$ and is almost lossless [26]. The structures studied in this section are composed of magnetic defect layer surrounded by 1D- MPCs. The examined magnetophotonic crystal consists of $\frac{\lambda}{2}$-thick defect layer (Ce:YIG) sandwiched between two 1D-MPC composed of 9 pairs of alternating $\frac{\lambda}{4}$ thick $\mathrm{SiO}_{2}$ and $\mathrm{Ce}: \mathrm{YIG}$ layers.

Fig. 2 shows the transmittance and the FR angle of this structure. The PBG covers the wavelength region from $1.35 \mu \mathrm{m}$ to $1.80 \mu \mathrm{m}$. In this region, the transmittance values and FR angles appear to be close to zero. In case of $\lambda_{\mathrm{s}}=1.55 \mu \mathrm{m}$, the transmittance value and FR angle are significantly increased and the resonance with $\mathrm{T}=0.54$ and $\theta_{\mathrm{F}}=41^{\circ}$ appears.

The enhancement of MO response of multilayered structures, such as FR angle, is obtained due to multiple reflections and their constructive interference in defect layer, which can be described by spatial field distribution. Fig. 3 shows the electric field distribution inside the structures which is localized in magnetic defect layer located in the middle of the structure.

As shown in Fig. 3b, a significant enhancement in the magnitude of the electric field is observed
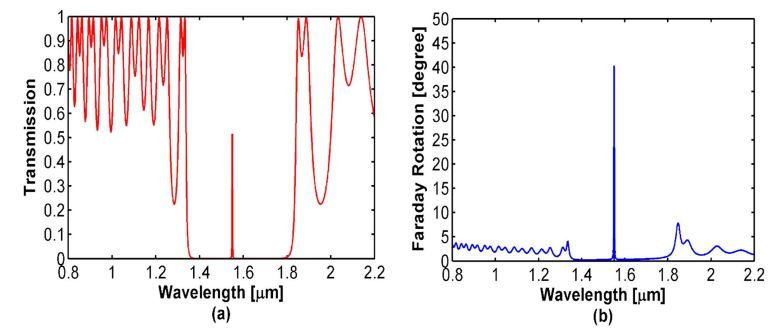

Fig. 2. (a) transmittance and (b) FR spectra of $(\mathrm{DM})^{9}(\mathrm{M})^{2}(\mathrm{MD})^{9}$ structure composed of $\mathrm{SiO}_{2}(\mathrm{D})$ and $\mathrm{Ce}: \mathrm{YIG}(\mathrm{M})$ layers.

in the cavity. The field amplitude in the defect layer is 2.2 times higher than that in the first layer. The constructive interference is confirmed by the field localization in the magnetic defect layer, followed by FR angle enhancement.

The thickness of the magnetic defect layer and repetition number of 1D-MPCs around it, are two effective parameters related to the optical and MO responses. Therefore, we measured the FR angles and transmittance values as a function of these parameters, which are shown in Fig. 4.

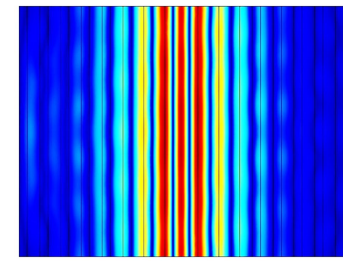

(a)

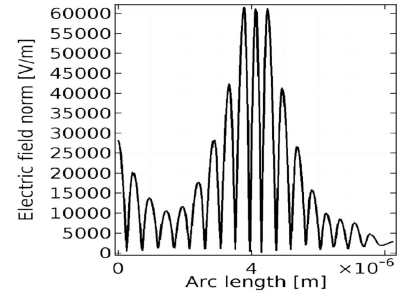

(b)
Fig. 3. (a) electric field distribution and (b) magnitude of electric field inside the magnetophotonic structure $(\mathrm{DM})^{9}(\mathrm{M})^{2}(\mathrm{MD})^{9}\left(\mathrm{D}=\mathrm{SiO}_{2}\right.$, $\mathrm{M}=\mathrm{Ce}: \mathrm{YIG})$.

In case of $0.5 \lambda_{\mathrm{s}}$-thick cavity, slight fluctuations in the transmittance and rotation angle, ranging from $\mathrm{m}=9$ to 14 are observed. The structures with transmittance values lower than 0.5 , indeed are inefficient for optical applications due to high losses. Accordingly, proper cases for optical applications occurred for the cavity thicknesses of $0.25 \lambda_{\mathrm{s}}, 0.5 \lambda_{\mathrm{s}}, 0.75 \lambda_{\mathrm{s}}, 0.875 \lambda_{\mathrm{s}}$.

The enhancement of transmittance and rotation angle happened in the defect mode inside the PBG 
or at the edges of the PBG region which depended on the thickness of defect layer. In the next step, we have considered the structures with magnetic defect layer sandwiched between the alternating $\mathrm{TiO}_{2}$ and Ce:YIG layers. Then, we have investigated the magnetophotonic structure with a repetition number $\mathrm{m}=9$ and $0.5 \lambda_{\mathrm{s}}$ optical thickness of the defect layer.

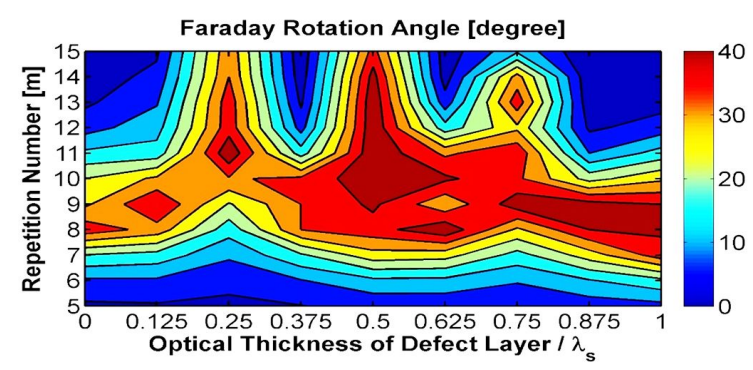

(a)

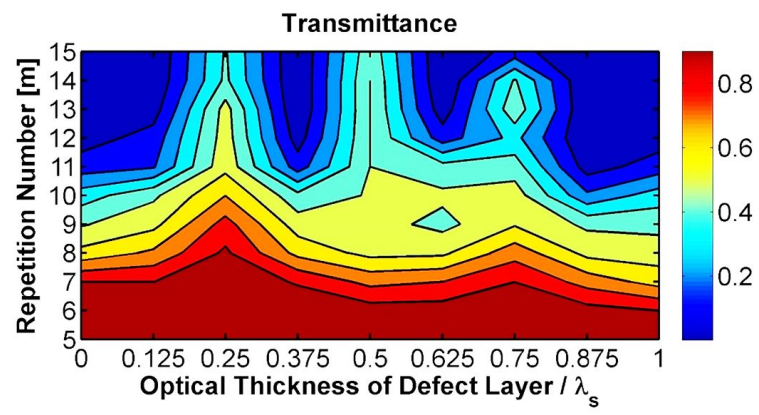

(b)

Fig. 4. (a) FR angle and (b) transmittance values of structures composed of $\mathrm{SiO}_{2}(\mathrm{D})$ and Ce:YIG(M) layers as a function of repetition number and optical thickness of defect layer.

The transmittance and its FR spectra are displayed in Fig. 5. As seen in Fig. 5, the transmittance value and the FR angle show a significant enhancement at $1.55 \mu \mathrm{m}$ inside the PBG. The FR value is equal to $\theta_{\mathrm{F}}=5.9^{\circ}$ and $\mathrm{PBG}$ is narrower than in the prior case.

For more accurate evaluation, the electric field spatial distribution has been simulated by FEM. The simulation results such as the magnitude of electric field are depicted in Fig. 6.

The width of PBG appears lower with weaker localization of light than in the prior case, which results from the low difference between the refractive indices of $\mathrm{TiO}_{2}$ and Ce:YIG materials. This leads
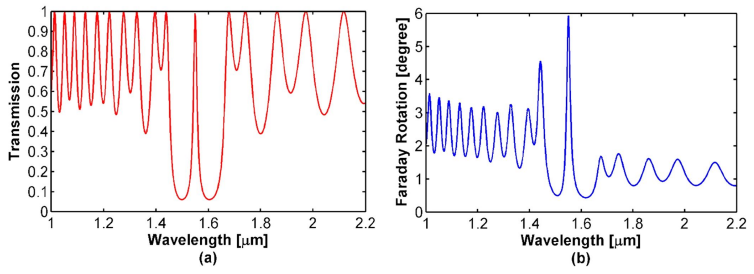

Fig. 5. (a) transmittance and (b) FR spectra of $(\mathrm{DM})^{9}(\mathrm{M})^{2}(\mathrm{MD})^{9}$ structure with $\mathrm{TiO}_{2}(\mathrm{D})$ and Ce:YIG(M) layers.

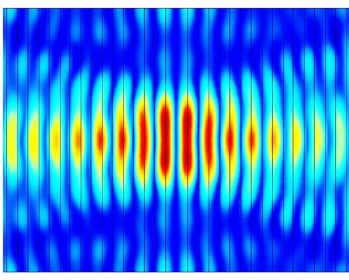

(a)

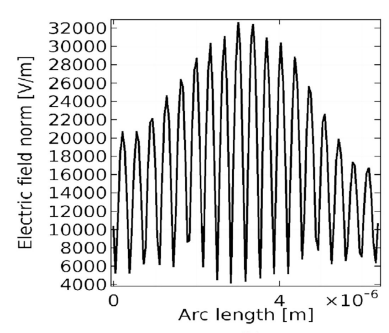

(b)
Fig. 6. (a) electric field distribution and (b) magnitude of electric field inside the magnetophotonic structure $(\mathrm{DM})^{9}(\mathrm{M})^{2}(\mathrm{MD})^{9}\left(\mathrm{D}=\mathrm{TiO}_{2}\right.$, $\mathrm{M}=\mathrm{Ce}: \mathrm{YIG})$.

to the reduction of MO properties of this structure, like for example the Faraday rotation angle. The FR angles of structures consisting of $\mathrm{TiO}_{2}$ and Ce:YIG, as a function of repetition number and the optical thickness of the defect layer, have been calculated, and they are shown in Fig. $7 \mathrm{a}$.

As can be seen in Fig. 7, in case of larger repetition number there is an enhancement in the FR angles for all of the magnetophotonic multilayers with different values of cavity thickness. A significant decrease in the magnitude of rotation angles has generally occurred due to poor field localization in the magnetic defect layer. The magnetophotonic crystals with $0.375 \lambda \mathrm{s}, 0.5 \lambda \mathrm{s}, 0.875 \lambda \mathrm{s}$ and $\lambda \mathrm{s}$ thickness of the defect layer appeared the most effective in terms of the highest FR angles. The transmittance values and rotation angles for different repetition numbers from 10 to 15 , are depicted in Fig. 7b and Fig. 7c for better analysis and comparison between the mentioned cases. The transmittance is decreasing for larger repetition numbers. For structures with $0.875 \lambda_{\mathrm{s}}$-thick and $\lambda_{\mathrm{s}}$ thick defect layer and $\mathrm{m}=15$, the transmittance 


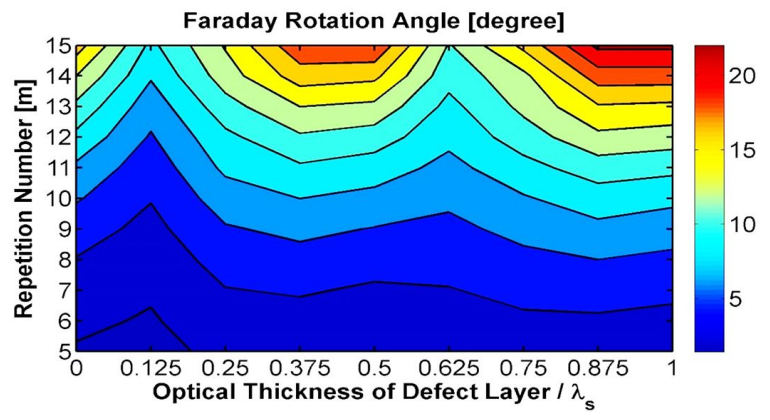

(a)

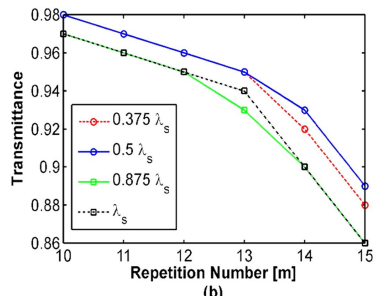

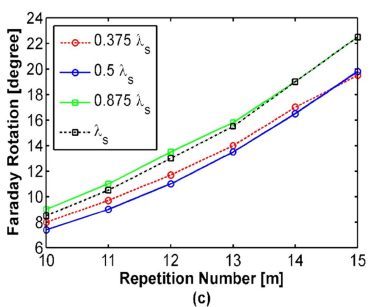

Fig. 7. (a) FR angle of structures composed of $\mathrm{TiO}_{2}$ (D) and Ce:YIG (M) materials as a function of optical thickness of defect layer and repetition number and the comparison of (b) transmittance and (c) FR angle versus the repetition number for the cases that were obtained at the higher values of rotation angle.

value and $\mathrm{FR}$ angle have reached $\mathrm{T}=0.86$ and $\theta_{\mathrm{F}}=22.5^{\circ}$. For $\mathrm{m}=15$, the rotation angles for $0.375 \lambda_{\mathrm{s}}$ and $0.5 \lambda_{\mathrm{s}}$ are equal to $19.5^{\circ}$ and $19.8^{\circ}$, respectively. A slight increase compared to the 0.875 $\lambda_{\mathrm{s}}$ and $\lambda_{\mathrm{S}}$ is observed in the transmittance values. A relatively significant decrease has been found in the MO response of the structures by comparing the rotation angles obtained in these multilayer structures with the values achieved for the previous structures. This appears to be a direct result of the weak field localization in the cavity located in the middle of the structures. The low difference between the refractive indices of the constituent materials of 1D-MPCs, would lead to such a behavior.

\subsection{Structures with dielectric Bragg mir- rors}

The structures with magnetic defect layer surrounded by dielectric Bragg mirrors are investigated in this section. Generally, two dielectric photonic crystals consisting of alternating $\mathrm{SiO}_{2}$ and $\mathrm{TiO}_{2}$ materials are placed at the right and left hand sides of the Ce:YIG defect layer. The optical thickness of each layer in these dielectric photonic crystals is a quarter wavelength.

At first, the structure with the repetition number equal to 7 and $0.5 \lambda_{\mathrm{s}}$ optical thickness of defect layer is studied. The transmittance and FR spectra of this magnetophotonic structure are shown in Fig. 8. The transmittance and rotation angle are enhanced at the defect mode wavelength $(1.55 \mu \mathrm{m})$ within the PBG. In this condition, $\mathrm{T}=0.65$ with $\theta_{\mathrm{F}}=37^{\circ}$.
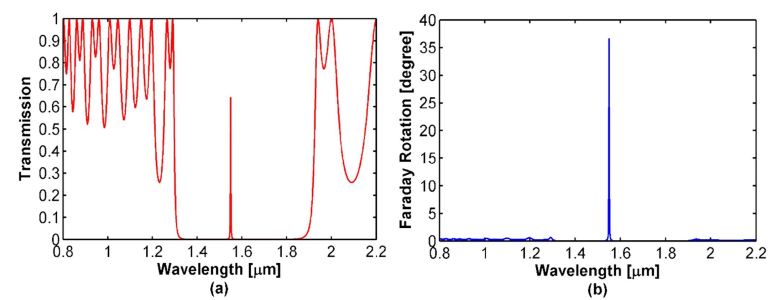

Fig. 8. (a) transmittance and (b) FR spectra of multilayer structure composed of magnetic defect layer surrounded by alternating layers of $\mathrm{SiO}_{2}$ and $\mathrm{TiO}_{2}$ materials.

As shown in Fig. 9, the electric field magnitude in the defect layer appears to be significantly higher than in the other layers, which indicates the strong field localization in the cavity due to the constructive interference of multiple reflections. Therefore, the enhancement significantly contributes to maximizing the MO properties of the structure, including the FR angle.

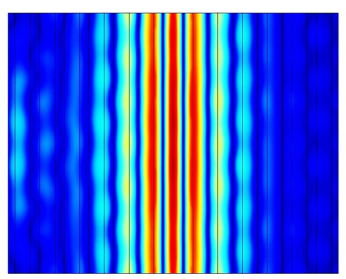

(a)

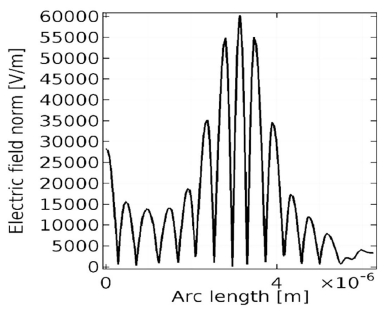

(b)
Fig. 9. (a) electric field distribution and (b) magnitude of electric field inside the magnetophotonic structure composed of magnetic defect layer surrounded by alternating layers of $\mathrm{SiO}_{2}$ and $\mathrm{TiO}_{2}$ materials. 
As it is shown in Fig. 10a, the magnetophotonic crystals show large rotation angles. For the structures with the cavity thicknesses of $0.375 \lambda_{\mathrm{s}}, 0.5$ $\lambda_{\mathrm{s}}, 0.625 \lambda_{\mathrm{s}}, 0.75 \lambda_{\mathrm{s}}$ and $0.875 \lambda_{\mathrm{s}}$, the FR angles have reached the values higher than 35 degrees. As seen in Fig. 10b and Fig. 10c, the rotation angle and transmittance for the structures with the half wavelength of cavity thickness and $\mathrm{m}=7$ are equal to $\theta_{\mathrm{F}}=37^{\circ}$ and $\mathrm{T}=0.65$.

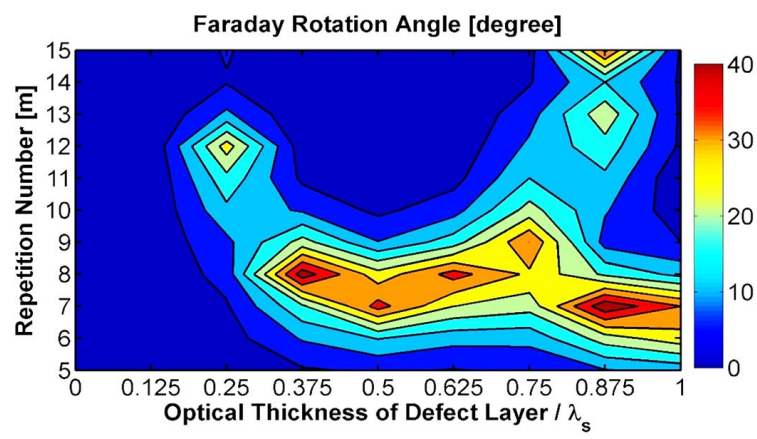

(a)
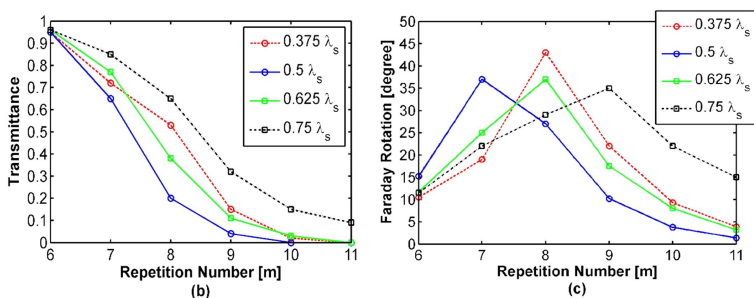

Fig. 10. (a) FR angle of structures composed of magnetic defect layer surrounded by alternating layers of $\mathrm{SiO}_{2}$ and $\mathrm{TiO}_{2}$ materials versus optical thickness of defect layer and repetition number; and the comparison of (b) transmittance and (c) FR angle versus the repetition number for the cases that were obtained at the higher values of rotation angle.

This situation seems to be beneficial for practical applications due to the high transmittance and rotation angle. For the magnetophotonic structure with $0.875 \lambda_{\mathrm{s}}$-thick defect layer, in case of $\mathrm{m}=15$, $\mathrm{T}=0.65$ and $\theta_{\mathrm{F}}=36^{\circ}$ have been obtained. These high rotations and transmittance, are optimal conditions for designing MO tools (Fig. 11).

\section{Conclusions}

We studied the magnetophotonic crystals with MO and dielectric Bragg mirrors. Two important

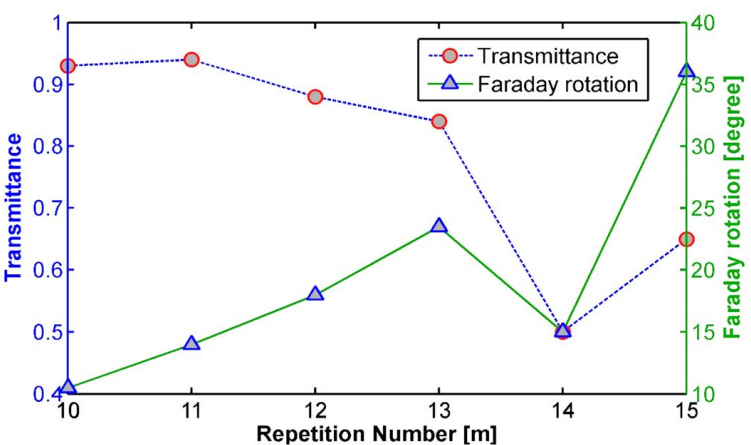

Fig. 11. Comparison of transmittance and FR angle of the structures composed of magnetic defect layer surrounded by alternating layers of $\mathrm{SiO}_{2}$ and $\mathrm{TiO}_{2}$ materials for $\mathrm{m}=10$ to $\mathrm{m}=15$.

parameters: the repetition number of Bragg mirrors around the defect layer and the optical thickness of the defect layer, which influence the transmittance and FR angle, have been considered. In the structures composed of $\mathrm{SiO}_{2}$ and $\mathrm{Ce}: \mathrm{YIG}$, due to a high difference between the refractive indices of constituent materials, the optical field was localized in the magnetic defect layer. This led to an increase in the MO responses of 1D-MPCs. The FR angles increased to values exceeding 35 degrees, despite the proper transmittance. The localization of the electric field in the magnetic defect layer was poor, due to the replacement of $\mathrm{SiO}_{2}$ with $\mathrm{TiO}_{2}$ where the rotation significantly decreased. In addition, we examined the magnetophotonic crystals with magnetic defect layer, surrounded by dielectric photonic crystals. In these structures, the electric field was localized mainly in the magnetic cavity. The FR angles of the structures were increased by multiple reflections of light in the cavity and constructive interferences. The MO multilayer structures with large rotation angles and proper transmittance can be used to design the MO devices such as sensors or the integrated photonic elements.

\section{References}

[1] Gaiyan B., Lijuan D., Shuai F., Zhifang F., Opt. Mater., 35 (2012), 252.

[2] Yablonovitch E., Phys. Rev. Lett., 58 (1987), 2059.

[3] John S., Phys. Rev. Lett., 58 (1987), 2486.

[4] Manzacca G., Paciotti D., Marchese A., MoreOlo M.S., Cincotti G., Photonic. Nanostruct., 88 (2007), 1. 
[5] Sugimoto Y., Tanaka Y., Ikeda N., Yang T., NAKAMURA H., ASAKAWA K., INOUE K., MARUYAMA T., MiYASHITA K., ISHidA K., WATANABE Y., Appl. Phys. Lett., 83 (2003), 3236.

[6] QIU M., Jaskorzynska B., Appl. Phys. Lett., 83 (2003), 1074.

[7] Inoue M., Arai K., Fujil T., Abe M., J. Appl. Phys., 85 (1999), 5768.

[8] Inoue M., Fujikawa R., BarysheV A., Khanikaev A., LiM P.B., Uchida H., AKTSipetrov O.A., Fedyanin A., Murzina T., Granovsky A., J. Phys. D. Appl. Phys., 39 (2006), 151.

[9] MurzinA T.V., KAPrA R.V., DOlgova T.V., FEDYANin A.A., AKTSIPETROV O.A., Nishimura K., Uchida H., Inoue M., Phys. Rev. B., 70 (2004), 012407.

[10] Kato H., Matsushita T., Takayama A., Egawa M., Nishimura K., Inoue M., J. Appl. Phys., 93 (2003), 3906.

[11] Fedyanin A.A., AKtsipetrov O.A., Kobayashi D., Nishimura K., UChidA H., INOUE M., IEEE. T. Magn., 40 (2004), 2850.

[12] Shimizu H., Miyamura M., Tanaka M., Appl. Phys. Lett., 78 (2001), 1523.

[13] Kato H., Inoue M., J. Appl. Phys., 91 (2002), 7017.

[14] Kato H., Matsushita T., Takayama A., Egawa M., Nishimura K., Inoue M., IEEE $T$. Magn., 38 (2002), 3246.

[15] Kahl S., Grishin A.M., Phys. Rev. B, 71 (2005), 205110.

[16] Goto T., Dorofeenko A.V., Merzlikin A.M., BarysheV A.V., Vinogradov A.P., Inoue M., Lisyansky A.A., Granovsky A.B., Phys. Rev. Lett., 101 (2008), 113902.
[17] Sakaguchi S., Sugimoto N., Opt. Commun., 162 (1999), 64.

[18] Sakaguchi S., Sugimoto N., IEEE J. Lightwave. Technol., 17 (1999), 1087.

[19] Kosobukin V.A., Solid State Commun., 139 (2006), 92.

[20] Inoue M., FujiI T., J. Appl. Phys., 81 (1997), 5659.

[21] Zvezdin A.K., Kotov V.A., Modern Magnetooptics and Magnetooptical Materials, CRC Press, Boca Raton, 1997.

[22] Jalali T., Hess Amodini M., Optik, 126 (2015), 3954.

[23] Koerdt C., Magneto-Spatial Dispersion Phenomena: Photonic Band Gaps and Chirality in Magneto-Optics (Doctoral dissertation), University of Konstanz, 2004. Retrieved from http: //www .ub.uni-konstanz.de/kops/volltexte/ 2004/1376/pdf/thesis-kops .pdf\&pli=1.

[24] Dyakov S.A., TolmacheV V.A., Astrova E.V., Tikhodeev S.G., Timoshenko V. Y., Perova T.S., (2010). Numerical methods for calculation of optical properties of layered structures, International Conference on Micro- and Nano-Electronics, Zvenigorod, Russian Federation, 2009, Russia: SPIE Digital Library.

[25] Bastos J.P.A., SAdowski N., Electromagnetic Modeling by Finite Element Methods, Marcel Dekker Inc., New York. Basel, 2003.

[26] Levy M., Yang H.C., Steel M.J., Fujita J., IEEE J. Lightwave. Technol., 19 (2001), 1964.

Received 2018-07-10 Accepted 2019-03-09 